\title{
User Based Content Management System to Analyze the E-Mail Reviews
}

\author{
Satinder Singh ${ }^{1}$, Dr. Amit Goel ${ }^{2}$ \\ ${ }^{1} \mathrm{M}$. Tech $4^{\text {th }}$ Sem, Department Of Computer Science and Engineering, SGT University,Gurgaon, India \\ ${ }^{2}$ Associate Professor \& HOD (Computer Science \& Engineering), Department of Computer Science And Engineering, SGT University, \\ Gurgaon, India
}

\begin{abstract}
Content management is characterized as a gathering of linkages between pages. Content Management frameworks (CMS) can be seen as information CMS stores and circulates the right content to the archives that may likewise contain creating, sequencing right learner at the ideal time. Additionally, CMS gives and content accumulation instruments, with a target to brought together capacity for little data lumps for simple "streamline the creation and organization of EMA recovery, update and appropriation. Initially created and utilized by the daily paper part of a CMS is the content itself. This paper proposed a new web application named TRACTOR that is content based review management systems
\end{abstract}

Keywords: Content Management frameworks (CMS), linkages between pages, TRACTOR,EMA,streamline

\section{Introduction}

The content in CMS is separated into and what is coming up short and plotting a photo for how they the physically displayable crude content and its - independently see content being taken care of after the CMS is put away format. The design itself comprises of two sections. These establishments should likewise recognize the one hand there is general data like "who" the essential clients and directors of the web asked for report sort. This data is called content are, for example, workforce, staff, organization and structure. Then again more definite data like understudies and empower a community exertion among them text styles, hues, and fringes and so on are required. This is known as the very effectively make those determinations. Higher showcase design and is generally put away in layouts or style instruction establishments must diagram their inside sheets [1].

The different media types, e.g. a printed Brochure and little lifecycle of overseeing data, spoke to by Website prompts the purported SSMM-rule (Single making, altering, distributed and documenting content, Source - Multiple Media). Notice that no is integral to any CMS execution. Foundations require just the partition of content and design (as expressed to diagram and characterize what sort of data is consistently above), additionally stockpiling of content in a media-made, the important approvers for the data, autonomous organization is expected to satisfy the solicitation of the where the data is distributed and when the SSMM-guideline. At the point when utilizing a CMS, the content itself is data ought to be chronicled. Normal data isolated into purported resources. Commonplace case for resources routinely distributed EMA by organizations is features and pictures [2].

Objectives of work:

- To audit the elements that has prompted the work on this anticipate.

- To examine the significance of open source and the part this plays in the accomplishment of this product based methodology.

- To discuss different arrangements which plan to take care of the same issues that address the show machine endeavors

- There is a decent correlation between these restrictive frameworks and the open arrangement that we have given.

- To present the unique subject issue on "Content management frameworks".

- To cover the examination of scope of themes from execution to interoperability, object-arranged database management frameworks, and exploration about addressing client needs.

\section{Background Studies}

Proposed approach is based on following studies:

Cornford and Pollock's (2002) endeavor to reveal the procedures required in putting colleges EMA is supplemented by different studies which concentrate, all the more particularly, on the issues encompassing site content management inside the setting of Higher Education [3].

Armstrong et al's. Exploration is reached out by Andrew Cox (2007) through bits of knowledge drawn from 15 semiorganized meetings of people in the UK college segment directed in 2004 [3]. These subjective bits of knowledge are investigated further in an overview of site content management crosswise over HEIs in the UK by Cox and Emmott (2007). As the most critical commitments to the comprehension of the procedures encompassing site content management in HEIs, notwithstanding the innovations included themselves, the suggestions and impediments of this exploration will now be considered inside and out [4].

The absence of joining at times found between IT support and different individuals from HEIs, Zabusky (1997) keeps up, is reflected in the physical area of IT workplaces, much of the time in peripheral ranges of the college; and examples 


\section{International Journal of Science and Research (IJSR) \\ ISSN (Online): 2319-7064 \\ Index Copernicus Value (2013): 6.14 | Impact Factor (2015): 6.391}

where individuals from IT support eat separated from different representatives [5].

The potential underestimation of those included in site management is likewise distinguished by Armstrong et al. who express that site administrators as often as possible report feeling "a level of expert separation inside their own particular associations". This is, maybe, on the grounds that their part is surprising - separating the limits between various callings and requesting a wide ability set including supervisor, plan, specialized specialization, bolster, research, advancement, preparing, and advertising. This outcomes, as expressed by Linford (1999), in an occupation part that "rapidly turns into an exercise in careful control of choosing which aptitudes to create, and picking a course in which to branch, while workload builds day by day with client desires and fast mechanical development." [6]

These bits of knowledge have suggestions for how people in site management are as of now upheld by HEIs and how this should be possible later on. Moreover, Cox's (2007) meeting discoveries demonstrate that the way web chiefs arrange inward legislative issues and attest the authenticity of their abilities and occupation part by partner themselves with various expert talks is to a great extent a result of individual decision. The assortment of these legitimizing talks is, maybe, itself, a result of the differing qualities of foundation and experience controlled by people in site management [7].

\section{Proposed Work}

\section{A. Implantation scenarios \\ Language and server used:}

MySQL is presently a standout amongst the most famous web scripting dialects, while Struts 2.0 is additionally the most predominant web server amongst minimal effort web hosts and designers running individual web servers. Another point of interest is that it is regular for MySQL and Struts 2.0 to be introduced close by each other. Struts 2.0 backing for per-catalog setups by means of an .htaccess document makes it simple for clients and engineers to tweak the earth without particular manager access; MySQL offers objectsituated programming includes that take into consideration the improvement of a MVC application that embraces huge numbers of the outline standards and components of a Rails system [8].

\section{Database used:}

Essentially, MySQL is an open-source Database Management System (DBMS) that is a standout amongst the most generally utilized Structured Query Language (SQL) executions for information stockpiling, close by big business level applications, for example, Oracle [9].

Application layout:

In order for each layer to logically handle requests and delegate responsibilities as best as possible. To begin with, TRACTOR gets an approaching HTTP ask for and summons the dispatcher. The dispatcher is a sort of metacontroller for TRACTOR that takes in a URL ask for and any POST information from the client, and does any work essential before the asked for controller is instantiated. The dispatcher first checks to ensure that TRACTOR's .htaccess record, arrangement document, and database are legitimately set up before conjuring the best possible controller. If TRACTOR is observed to be not set up, and then the dispatcher consequently overlooks the URL ask for and conjures the setup controller to start the best possible stride of the set up procedure. If the application is set up, then the dispatcher parses the URL ask for and summons the fitting controller [10].

\section{B. Implementation modules}

1) User

- Signup: the super-client has control concerning regardless of whether the information exchange sheet is accessible to the general population, yet this discourse includes a straightforward route for clients and managers to include new records [11].

- Login: the present client is provoked for their qualifications. Their username and secret word are asked for and after that validated.

- Logout: This is another bit of critical usefulness that is without a XHTML view. The capacity is straightforward; it deletes the majority of the session information as of now set and alarms the client that they have logged out. The client is then diverted to the landing page.

- Show: now clients are allowed to see a solitary client and the greater part of the information that is connected with that client.

\section{2) SMS}

- Add: If a client is signed in and has adequate benefits to include a message, they are given a structure that permits them to fill in meta information.

- Edit: This perspective is much similar to the include view. It is outwardly comparable aside from it comes populated with information connected with the message.

- Remove: There is no discrete XHTML view connected with the evacuate usefulness; however an alarm is passed onto the executive who expels the message.

- Show: Tags are an advantageous technique for clients to connect data to their messages. These words permit the viewer to all the more effortlessly pick through the content that is being exhibited. Despite the channel or message, a viewer can instantly enter in on some particular term and have the capacity to recover an EMA of content pertinent to that term [12].

\section{3) DAO}

- Add: With this exchange, a head can include another channel and partner a couple bits of Meta information. We presently permit titles, portrayals, labels and symbols to be connected to a channel.

- Edit: The channel alter structure is much similar to that of the include discourse, yet this structure comes prepopulated with the information from a current channel. Any bit of information that can be included can likewise be altered.

- Remove: Nothing should be shown for the expel activity. Rather, when a manager goes to expel a channel, we essentially alarm them that their channel has been 


\section{International Journal of Science and Research (IJSR) \\ ISSN (Online): 2319-7064 \\ Index Copernicus Value (2013): 6.14 | Impact Factor (2015): 6.391}

evacuated and after that we forward them to the default channel page.

- Show: This page gives the client an approach to see Meta information and to download the content itself. It additionally serves as a hopping point to the channels that the content has a place with and to the labels that have been connected with the content.

- Download: While the download segment of every message does not have a conventional HTML see, this is the most vital part of the application. This page diverts the client to a URI where the message document can be downloaded.

\section{Results}

Results of our proposed technology will be like following below figures:

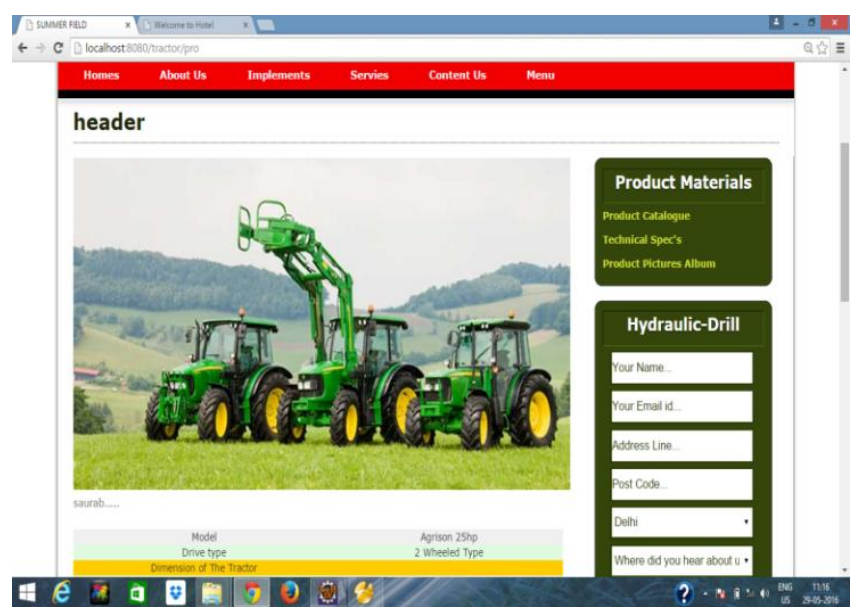

Figure 1: Home page for tractor website named as SUMMER FIELD

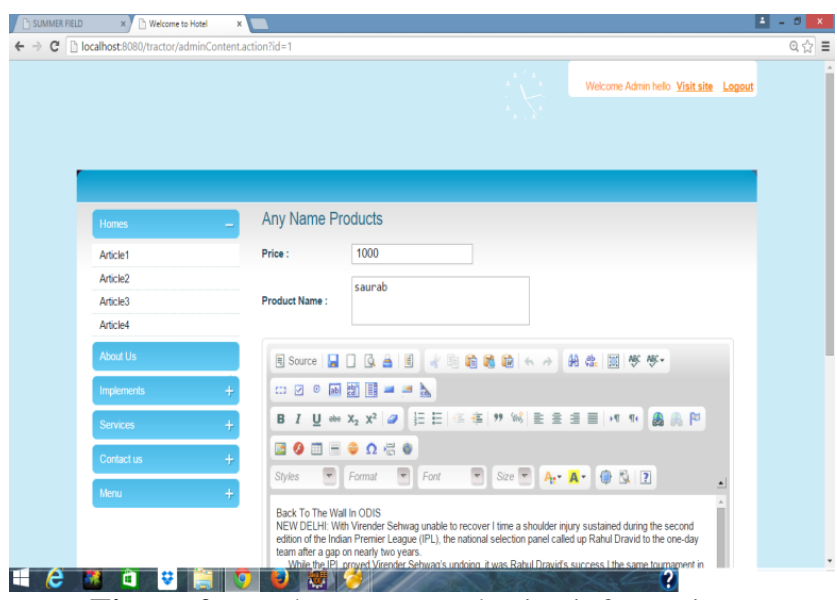

Figure 2: Product name and price information page

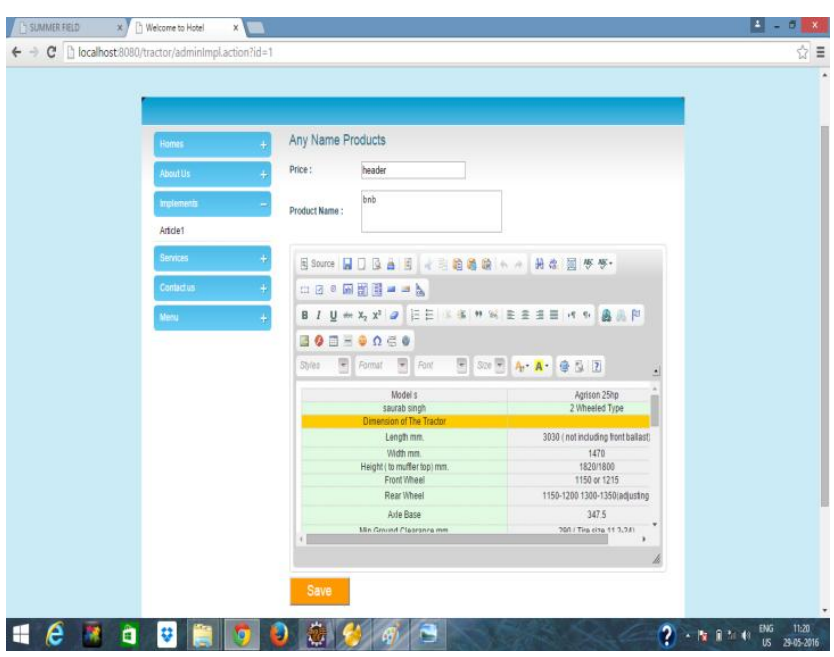

Figure 3: Under implements tab and product details

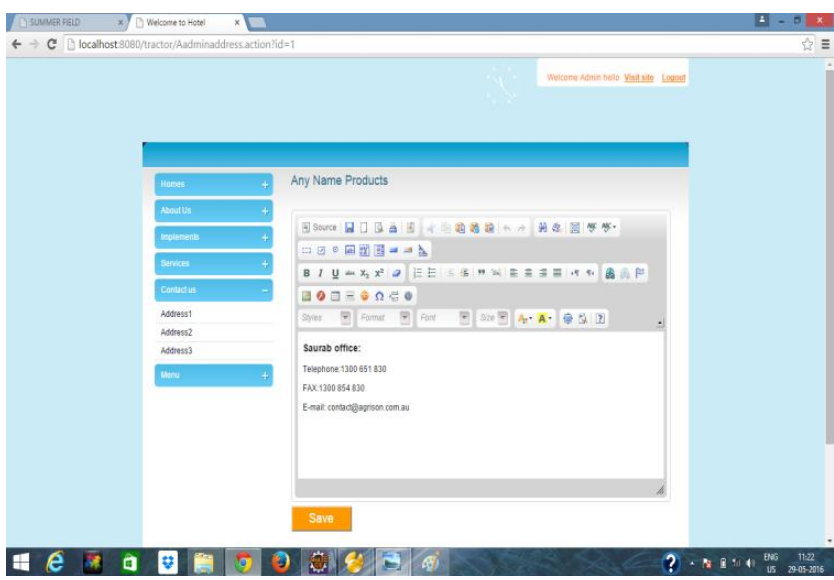

Figure 4: User details under contact us tab

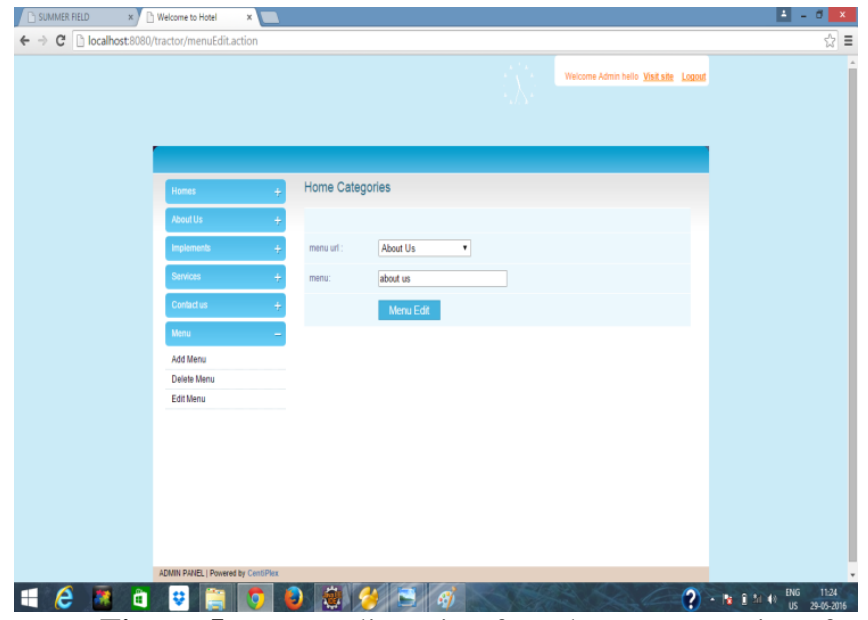

Figure 5: Menu edit option from home categories of project

\section{Volume 5 Issue 6, June 2016 www.ijsr.net}




\section{International Journal of Science and Research (IJSR) \\ ISSN (Online): 2319-7064}

Index Copernicus Value (2013): 6.14 | Impact Factor (2015): 6.391

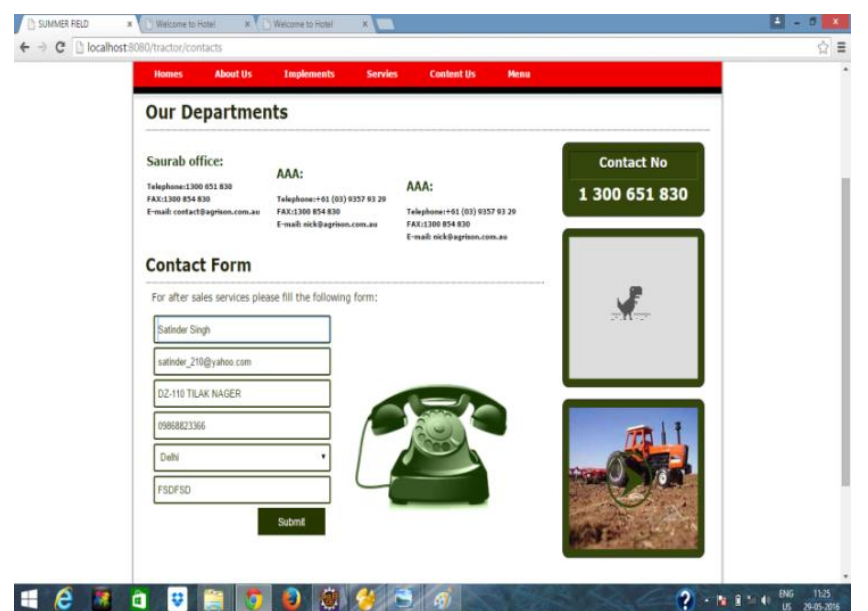

Figure 6: Departments page for Seller details and contact form

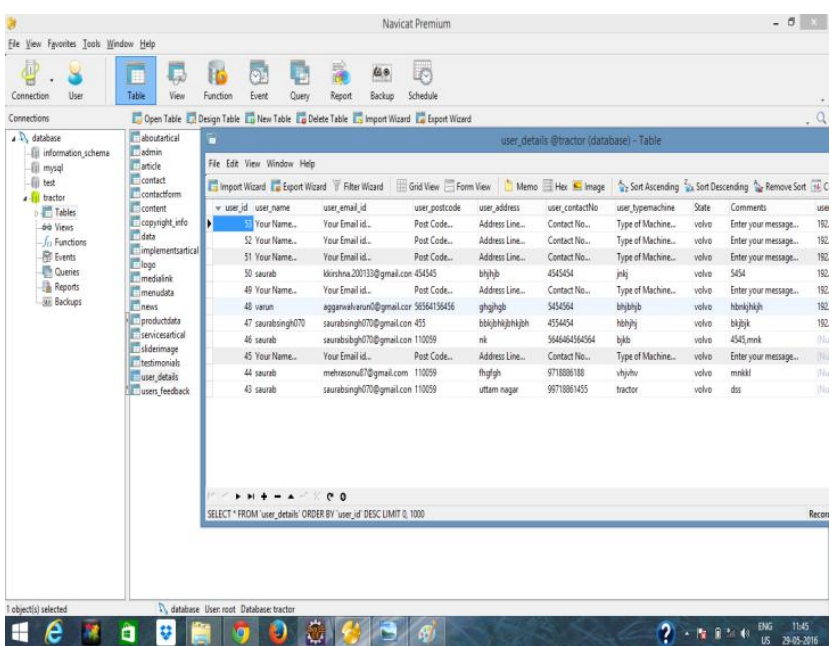

Figure 7: Navicat premium window showing database setup for user details wizard

\section{Conclusions}

Web message is one of the quickest developing data mediums in the public eye today. By looking at the engineering and ruins of the past TRACTOR, we could astutely outline a steady successor that empowers message makers to effortlessly distribute their work on the Internet. We have made a strong engineering that is anything but difficult to use for distributers, viewers, fashioners, and designers alike. The new TRACTOR permits distributers to share amazing message all alone terms, and without unique specialized learning.

We trust this new form of TRACTOR appreciates the same measure of ubiquity as its forerunner. Our group feels that this product can be a valuable device and has the likelihood to change the present environment for Internet message.

\section{References}

[1] Alain Andrieux, Karl Czajkowski, Asit Dan, Kate Keahey, Heiko Ludwig, Toshiyuki Nakata, Jim Pruyne, John Rofrano, Steve Tuecke, and Ming Xu. Web Services Agreement Specification. https://forge.gridforum.org, 92006.
[2] A. Avizienis, J. Laprie, and B. Randell. Fundamental Concepts of Dependability. Research Report N01145, LAAS-CNRS, April 2001, 42001.

[3] Tim Banks. Web Services Resource Framework (WSRF) - Primer v1.2. Internet, 5 2006. Committee Draft 02 - 23 May 2006.

[4] Don Box, Erik Christensen, Francisco Curbera, Donald Ferguson, Jeffrey Frey, Marc Hadley, Chris Kaler, David Langworthy, Frank Leymann, Brad Lovering, Steve Lucco, Steve Millet, NirmalMukhi, Mark Nottingham, David Orchard, John Shewchuk, EugèneSindambiwe, Tony Storey, SanjivaWeerawarana, and Steve Winkler. Web Services Addressing (WSAddressing). Technical report, The World Wide Web Consortium (W3C), 2004. W3C Member Submission 10 August 2004.

[5] T. Banks, A. Djaoui, S. Parastatids, A. Mani, S. Tuecke, K. Czajkowski, I. Foster, J. Frey, S. Graham, C. Kesselman, T. Maguire, T. Sandholm, D. Snelling, and P. Vanderbilt. Open Grid Services Infrastructure (OGSI) Version 1.0 http://www.ggf.org/documents/GFD.15.pdf, 62003.

[6] Keith Ballinger, David Ehnebuske, Christopher Ferris, Martin Gudgin, Canyang Kevin Liu, Mark Nottingham, and Prasad Yendluri. Basic Profile Version 1.1. http://www.ws-i.org.

[7] MalteBiß. Componentization and Orchestration of Content Management Services. Master's thesis, Universität Hamburg, 2007.

[8] Kenneth Chiu, MadhusudhanGovindaraju, and Randall Bramley. Investigating the Limits of SOAP Performance for Scientific Computing. In HPDC, pages 246-254. IEEE Computer Society, 2002.

[9] Frank Cohen. FastSOA: The way to use native XML technology to achieve Service Oriented Architecture governance, scalability, and performance (The Morgan Kaufmann Series in Data Management Systems). Morgan Kaufmann Publishers Inc., San Francisco, CA, USA, 2006.

[10] Shiping Chen, Bo Yan, John Zic, Ren Liu, and Alex $\mathrm{Ng}$. Evaluation and Modeling of Web Services Performance. icws, 0:437-444, 2006.

[11] Ian Foster and Carl Kesselman. The Grid: Blueprint for a New Computing Infrastructure. Morgan Kaufmann Publishers Inc., San Francisco, CA, USA, 1999.

[12]Ian Foster, Carl Kesselman, Jeffrey M. Nick, and Steven Tuecke. The Physiology of the Grid: An Open Grid Services Architecture for Distributed Systems Integration, 2002. 